\title{
Improved early outcome for end-stage dilated cardiomyopathy in children
}

\author{
Anne-Marie McMahon, MRCPCH \\ Carin van Doorn, FRCS (C/Th) \\ Michael Burch, FRCP \\ Pauline Whitmore, RSCN \\ Sophie Neligan, RSCN \\ Philip Rees, FRCP \\ Rosemary Radley-Smith, FRCP \\ Allan Goldman, FRCPCH \\ Katherine Brown, MRCP \\ Gordon Cohen, MD \\ Victor Tsang, FRCS \\ Martin Elliott, MD, FRCS \\ Sponsored by Marc R. de Leval, MD, FRCS
}

From the Great Ormond Street Hospital for Children National Health Service Trust, London, United Kingdom.

Research at the Institute of Child Health and Great Ormond Street Hospital for Children NHS Trust benefits from Research and Development funding received from the NHS Executive.

Received for publication May 2, 2003; revisions requested July 16, 2003; accepted for publication July $31,2003$.

Read at the Eighty-third Annual Meeting of The American Association for Thoracic Surgery, Boston, Mass, May 4-7, 2003.

Address for reprints: Carin van Doorn, FRCS (C/Th), Cardiothoracic Unit, Cardiac Wing, Level 2, Great Ormond Street Hospital for Children NHS Trust, Great Ormond Street, London WC2A 3JH, United Kingdom (E-mail: vandoc@gosh.nhs.uk).

J Thorac Cardiovasc Surg 2003;126:1781-7

Copyright $\odot 2003$ by The American Association for Thoracic Surgery

$0022-5223 / 2003 \$ 30.00+0$

doi:10.1016/j.jtcvs.2003.07.029
Objective: To review the impact of management changes on the early outcomes of end-stage dilated cardiomyopathy in children.

Methods: We conducted a retrospective study of all consecutive children with end-stage dilated cardiomyopathy who received hospital treatment since 1992. Over the past 3 years the following management changes were made: (1) more aggressive use of mechanical cardiac assistance; (2) high priority listing for transplantation; and (3) ABO incompatible transplants for infants. Outcomes for 46 patients admitted between 1992 and 1999 (group I) were compared with 53 patients between 2000 and March 2003 (group II).

Results: In group I, 12 (26\%) patients received mechanical support with recovery in 3 and transplantation in 5 (1 died). In group II, 19 (36\%) patients received extracorporeal membrane oxygenation, with recovery in 5 and transplantation in 12 (all survived). The use of mechanical assistance was associated with high morbidity related to bleeding, end-organ failure, and long-term mechanical ventilation. Five patients in group II received ABO incompatible transplants and all survived. There have been no episodes of rejection or need for increased immunosuppressive therapy. Hospital mortality has been significantly reduced (group I, 37\% vs group II, $11 \% ; P<.05)$.

Conclusions: Recent refinements in the management of end-stage dilated cardiomyopathy in children have significantly reduced early mortality. Identification of markers of early myocardial recovery and development of mechanical devices for longer term and more physiologic support are essential to achieve further improvements in outcome.

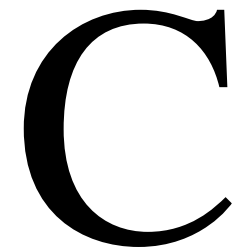
ardiac transplantation is still the only definitive treatment available for end-stage dilated cardiomyopathy (EDCM) in children, and donor organ availability remains an important limiting factor. A recent audit of the UK Pediatric Transplant Database, however, revealed that this was not due to an absolute shortage of donor organs but to a mismatch between timing of availability of the donor heart and recipient demand. ${ }^{1}$ Thus, in 1996/1997, 20 children died on the 
waiting list for cardiac transplantation, while during the same period 59 pediatric hearts were not used because no suitable recipient was available. It was hypothesized that if the 20 patients could have been kept alive until the next suitable unused heart became available, all but 2 could have had transplantation within a median time of 63 days, and in 3 patients the waiting time would have been less than 1 week. To make more effective use of the donor pool, a program for mechanical cardiac assistance as a bridge to transplantation was initiated in 1998. This program was approved by the Research Ethics Committee of the Institute of Child Health/Great Ormond Street Hospital for Children National Health Service (NHS) Trust.

Great Ormond Street Hospital for Children NHS Trust is a supraregional center for extracorporeal membrane oxygenation (ECMO), and because of this experience ECMO was used to bridge the initial patients. However, since the time on mechanical support was estimated (1998) to last up to several months, we moved to paracorporeal ventricular assist devices (VADs) with the expectation that this would allow cardiac support with recovery of end-organ function outside intensive care. ${ }^{2}$ Unfortunately, patients with a VAD remained dependent on mechanical ventilation and inotropic agents and had significant complications. We therefore changed back to ECMO (1999), which has been used exclusively for the past 3 years.

In 2000, all transplant centers in the United Kingdom agreed on high priority listing for patients in urgent need of transplantation (equivalent to United Network for Organ Sharing [UNOS] status 1A), including children on mechanical cardiac support. Each of the two United Kingdom pediatric transplant centers can only list one priority patient at any one time, and the first compatible organ is offered to this patient.

More recently, following the pioneering work of the Toronto group, ${ }^{3}$ we started to perform heart transplantations with ABO-incompatible donors. ABO-incompatible transplants can be performed without the occurrence of hyperacute rejection in young children, because they do not yet produce antibodies to major blood group antigens. We considered children less than 18 months for ABO-incompatible transplant if anti-A or anti-B isohemagglutinin titers were $1: 16$ or less.

This article reviews the impact of these recent changes (since 2000) on the early outcome of EDCM in children in our institution.

\section{Patients and Methods Patients}

The records of all patients with EDCM treated under our care between January 1, 1992, and March 31, 2003, were reviewed. The definition of EDCM in children was patients younger than 16 years with dilated cardiomyopathy requiring hospital admission with inotropic support with or without mechanical support.
Patients were divided into 2 groups: Group I (patients before the management changes, January 1992 to December 1999) included 46 children aged between 1 month and 16.1 years (median 2.1 years). Group II (after the management changes, January 2000 to March 2003) included 53 children aged between 7 days and 16.0 years (median 2.0 years).

\section{Indication for Use of Mechanical Support}

Mechanical bridging to transplantation was considered in patients with EDCM who showed hemodynamic deterioration with inotropic support, with or without mechanical ventilation. Children less than 1 year old were not considered for bridge to transplantation because the scarcity of small donor hearts in the United Kingdom made it unlikely that an organ would be available during the period on mechanical support. However, if recovery of cardiac function was thought possible, mechanical bridging to recovery was considered. Severe neurologic injury and parental refusal were also contraindications for mechanical support.

The timing of initiating mechanical support is difficult in patients with severe heart failure who sometimes decompensate suddenly. Patients were therefore closely observed in the high dependency unit for signs of deteriorating renal, hepatic, and gastrointestinal function, alteration of mental state, or poor peripheral perfusion. Other indications for ECMO included the development of cardiac arrhythmias and escalating inotropic requirement. Sixty-six percent of patients received a minimum of 2 inotropic agents. Pulmonary artery catheters were not used to assess cardiac performance. More recently, we had a clear-fluid primed ECMO circuit on standby so that in case of cardiac deterioration ECMO could be instituted immediately.

\section{Mechanical Assist Devices}

ECMO. A standardized ECMO circuit including a roller pump and appropriately sized tubing and cannulas for patient weight was used. ECMO cannulation was routinely performed on the intensive care unit and typically involved open cannulation of the right common carotid artery and internal jugular vein through a 2 to $3-\mathrm{cm}$ skin crease incision in the neck. In patients over $10 \mathrm{~kg}$, additional venous drainage was often necessary, and a venous cannula was placed in a femoral vein by a percutaneous Seldinger technique. ECMO was initially commenced aiming at pump flows of at least $100 \%$ of calculated resting cardiac output for body size and adjusted according to body perfusion, arterial oxygenation, and mixed venous oxygenation. Inotropic agents were weaned as tolerated and hypertension was treated with vasodilators. If renal support was required, a hemofilter was built into the ECMO circuit. Decompression of the left side of the heart was routine and performed by balloon or blade septostomy under fluoroscopy and ultrasound guidance in the catheter laboratory, usually within 24 hours of initiating ECMO. Patients were nursed sedated and ventilated on the intensive care unit. Anticoagulation for ECMO consisted of intravenous heparin 5 to $25 \mathrm{U} \cdot \mathrm{kg}^{-1} \cdot \mathrm{h}^{-1}$ maintaining an activated clotting time between 160 and 180 seconds.

VAD. The Medos-HIA VAD (Stolberg, Germany) is a paracorporeal and pneumatically driven device that is available in a range of sizes of pumping chambers and cannulas. Left ventricular assist devices (LVAD) range from $10 \mathrm{~mL}$ stroke volume for infants to $80 \mathrm{~mL}$ for adults, with $10 \%$ reduced volume for right ventricular support. Device placement was carried out in the op- 
TABLE 1. Mechanical cardiac support

\begin{tabular}{lcc}
\hline & Group I: & Group II: \\
12 patients & 19 patients \\
\hline Age & $1.8 \mathrm{y}-16.1$ y $(10.0 \mathrm{y})$ & $7 \mathrm{~d}-16.0$ y $(3.9 \mathrm{y})$ \\
VAD & 5 & 0 \\
ECMO & 7 & 19
\end{tabular}

$V A D$, Ventricular assist device; ECMO, extracorporeal membrane oxygenation.

erating theater via median sternotomy and on cardiopulmonary bypass (CPB). For placement of the LVAD, the left atrium was cannulated for device inflow and the ascending aorta for outflow. RVAD cannulation was via the right atrium and pulmonary artery. After device placement, CPB was discontinued with reversal of heparin and transfusion of blood products as necessary to achieve a normal coagulation profile. Intravenous heparin was commenced after 4 hours aiming at an activated clotting time of 160 to 180 seconds.

Mechanical circulatory support was used in 12 of 46 (26\%) patients in group I and in 19 of 53 (36\%) patients in group II. The devices used for assistance are summarized in Table 1. Between September 1998 and July 1999, 4 patients were supported on the Medos-HIA. One patient received an LVAD, and in the remaining 3 patients, both ventricles were supported (BIVAD). A further patient was placed on a Berlin Heart BIVAD (Berlin Heart, Berlin, Germany) in another institution by the Great Ormond Street team and then referred to our institution. The Berlin Heart operates along the same principles as the Medos-HIA VAD. The remaining patients all received ECMO.

\begin{abstract}
ABO-Incompatible Transplantation
We followed the guidelines for ABO-incompatible transplantation previously described by West and coworkers. ${ }^{3}$ In short, patients underwent standard blood typing, and serum was tested for anti-A and anti-B antibodies with standard agglutination tests with erythrocytes from a known blood type. The blood type of the donor was obtained via the transplant coordinator. All cellular blood products given during or after transplantation were either blood type $\mathrm{O}$ (which contains no A or B antigens) or the recipient's blood type. All plasma products were type $\mathrm{AB}$ (or the donor's blood type if the recipient was type $\mathrm{O}$ ) and therefore had few or no exogenous anti-A or anti-B antibodies. Platelets were donor group A or B type specific, as type $A B$ is not available in the United Kingdom. At the initiation of $\mathrm{CPB}$, exchange transfusion was performed, up to 3 times the estimated circulating blood volume of the recipient. Blood was drained via the venous limb of the bypass circuit and replaced with an equal volume of bank blood and plasma from type AB blood. Discarded blood was separated into plasma and red-cell fractions with a commercial cell salvage system, the plasma was discarded, and the red cells were returned to the patient.
\end{abstract}

\section{Results}

\section{Outcome After Mechanical Support}

The outcomes of the 31 patients who received mechanical assistance for EDCM are given in Table 2. Three patients
TABLE 2. Outcomes following mechanical cardiac support

\begin{tabular}{lccccc}
\hline & \multicolumn{2}{c}{ Group I (12 patients) } & & Group II (19 patients) \\
\cline { 2 - 3 } & $\begin{array}{c}\text { No. of } \\
\text { patients }\end{array}$ & $\begin{array}{c}\text { Days on } \\
\text { support }\end{array}$ & & $\begin{array}{c}\text { No. of } \\
\text { patients }\end{array}$ & $\begin{array}{c}\text { Days on } \\
\text { support }\end{array}$ \\
\hline Recovery & 3 & $4-10(7)$ & 5 & $6-12(9)$ \\
Transplant & 5 & $0.5-9(6)$ & 12 & $2-21(7)$ \\
Support withdrawn & 4 & $7-12(10)$ & 2 & $8-11$ \\
Overall mortality & $5 / 12(42 \%)$ & & $2 / 19(11 \%)$ & \\
\hline
\end{tabular}

(25\%) in group I and 5 (26\%) in group II showed improvement of myocardial function on echocardiography within 48 hours and were successfully weaned from support. Seven of $8(87.5 \%)$ patients bridged to recovery are alive at follow-up from 5 to 81.5 months ( 23 months). One patient died suddenly at home after 19 months. One patient in group II with Epstein-Barr viral myocarditis had a further episode of severe heart failure 20 months later. He had a second ECMO run followed by successful cardiac transplantation. This patient appears in the statistics twice.

Five (42\%) patients in group I and $12(63 \%)$ in group II underwent cardiac transplantation. The patient on the Berlin Heart underwent successful transplantation on day 5 by the Great Ormond Street Hospital team at the referring hospital and was subsequently transferred to our institution for postoperative care. There was 1 hospital death. Acute respiratory distress syndrome developed in a patient in group I and necessitated posttransplant ECMO. She died of multiorgan failure 8 days after cardiac transplantation. There was 1 late death at 50 months from rejection related to noncompliance with medication. The long-term survivors have now been observed for 5 weeks to 108 months (17 months).

Mechanical support was withdrawn in 4 patients in group I. Large cerebral infarcts developed in 2 patients during support on the Medos VAD. Multiorgan failure developed in 2 patients on ECMO, including 1 in whom the parents declined transplantation and requested withdrawal of mechanical support. ECMO support was withdrawn in 2 patients in group II. One patient had severe neurologic injury and renal failure. He had been ventilated for 4 days before ECMO and required cardiopulmonary resuscitation 24 hours before ECMO was commenced. The other patient was a 7-day-old child with cardiorespiratory collapse who was placed on ECMO as a bridge to recovery. A diagnosis of enteroviral myocarditis was made. Progressive multiorgan failure developed and, in agreement with the parents, support was withdrawn on day 8 .

The use of mechanical cardiac assistance was associated with a large number of complications. The 4 patients on the Medos VAD all required reexploration for bleeding and tamponade and had ongoing requirements for inotropic support and mechanical ventilation. Two had irreversible brain 
TABLE 3. Outcomes following high priority listing

\begin{tabular}{lccl}
\hline $\begin{array}{l}\text { Pre-transplant } \\
\text { support }\end{array}$ & No. of patients & $\begin{array}{c}\text { Days on high } \\
\text { priority list }\end{array}$ & Outcome \\
\hline Medical treatment & 8 & $3-39(6)$ & $\begin{array}{l}\text { Died 4 } \\
\text { Transplant 4 }\end{array}$ \\
ECM0 & 11 & $2-21(6)$ & Transplant 11
\end{tabular}

Fifteen of $19(79 \%)$ were offered organs. All survived. ECMO, Extracorporeal membrane oxygenation.

TABLE 4. ABO mismatch transplant: Donor and recipient blood groups.

\begin{tabular}{cc}
\hline Recipient blood group & Donor blood group \\
\hline $\mathrm{B}+$ & $\mathrm{A}-$ \\
$0+$ & $\mathrm{A}+$ \\
$\mathrm{A}+$ & $\mathrm{AB}+$ \\
$\mathrm{A}+$ & $\mathrm{AB}+$ \\
$0+$ & $\mathrm{A}+$ \\
\hline
\end{tabular}

injury, and renal support was required in 2 patients. In contrast, the patient on the Berlin Heart had no severe bleeding or renal problems. In the 26 patients who received ECMO there were no significant bleeding problems. However, the following problems were observed: prolonged ventilation ( $>5$ days) after transplant in 9 patients (range 7-48 days, median 24), of whom 6 required a tracheostomy; renal failure necessitating peritoneal dialysis or hemofiltration (10 patients); medical treatment for withdrawal of sedation (8 patients); and resolving right hemiparesis (1 patient).

\section{High Priority Listing for Transplantation}

Nineteen patients in group II were put on the high priority list, including 11 of 12 ECMO patients (Table 3). One ECMO patient could not be listed because another patient had already been priority listed. Fifteen patients had transplants and all survived. The 4 patients who died included a 7-month old child who had an acute respiratory arrest after waiting for 39 days. Two patients had cardiac arrest on days 6 and 10, respectively. Multiorgan failure developed in 1 patient 3 days after listing; the parents requested no further active intervention.

\section{ABO Incompatible Transplantation}

Six patients were listed for $\mathrm{ABO}$ mismatch transplantation, 2 of whom were high priority listed, including 1 patient on ECMO. All had transplants. One patient with blood group A received a compatible donor heart within 24 hours. Five patients underwent $\mathrm{ABO}$ incompatible transplantation after 5 to 87 days (13 days). The blood groups of the donors and recipients are detailed in Table 4 . None of the patients had hyperacute rejection. All survived and have now been fol-
TABLE 5. Effect of management strategy on hospital survival for end-stage dilated cardiomyopathy in children

\begin{tabular}{|c|c|c|c|c|c|}
\hline & & \multicolumn{2}{|c|}{ Group I } & \multicolumn{2}{|c|}{ Group II } \\
\hline & & $\begin{array}{c}\text { No. of } \\
\text { patients }\end{array}$ & $\begin{array}{l}\text { No. } \\
\text { died }\end{array}$ & $\begin{array}{c}\text { No. of } \\
\text { patients }\end{array}$ & $\begin{array}{l}\text { No. } \\
\text { died }\end{array}$ \\
\hline \multirow[t]{2}{*}{$\begin{array}{l}\text { Medical } \\
\text { therapy }\end{array}$} & $\begin{array}{l}\text { Medical treatment } \\
\text { only }\end{array}$ & 20 & 11 & 16 & 4 \\
\hline & Transplant & 14 & 1 & 18 & 0 \\
\hline \multirow[t]{3}{*}{$\begin{array}{l}\text { Mechanical } \\
\text { support }\end{array}$} & Recovery & 3 & 0 & 5 & 0 \\
\hline & Transplant & 5 & 1 & 12 & 0 \\
\hline & Support withdrawn & 4 & 4 & 2 & 2 \\
\hline Total & & 46 & $17^{*}(37 \%)$ & 53 & $6^{*}(11 \%$ \\
\hline
\end{tabular}

lowed up for 2 to 20 months (5 months). The 2 patients with detectable anti-A or anti-B antibodies before transplantation have remained antibody-negative at most recent follow-up. Only 1 other patient had low antibody titers (1:1) to the incompatible graft. Maintenance immunosuppressive therapy includes tacrolimus and either azathioprine or mycophenolate mofetil. No patient is on long-term steroids. There have been no episodes of humoral rejection.

Table 5 summarizes the overall effect of the change in EDCM on early outcome. In the most recent treatment group, hospital survival has significantly improved (group I: 29/46 patients (63\%) versus group II: $47 / 53$ patients (89\%), $P<.05)$.

\section{Comment}

Our recent experience with mechanical bridging to transplantation compares favorably with the reported pediatric literature. Kirshbom and coworkers ${ }^{4}$ reported on 31 children who underwent bridging to cardiac transplantation with ECMO, either because of postcardiotomy failure (17) or cardiomyopathy, myocarditis, or other reasons (14). Six were weaned and survived to discharge and 12 went on to transplantation. The authors did not comment on hospital survival but reported $83 \%$ survival at 1 year. More recently, Gajarski and associates ${ }^{5}$ published a series of 145 children supported on ECMO, 21 of whom were listed for transplantation. The diagnosis was postcardiotomy failure in 17, myocarditis in 2, and myopathy in a further 2 patients. Twelve patients underwent transplantation and 10 survived to discharge.

Despite excellent survival after bridging in our patients, a large number of difficulties remain associated with mechanical cardiac assistance in children. In patients supported with the Medos VAD who underwent cannulation on CPB, bleeding was a major problem. Furthermore, the cerebral infarcts were a cause for concern. Interestingly, the single patient supported with the Berlin Heart had no bleeding or neurologic problems. In the ECMO group who underwent 
peripheral cannulation, bleeding was not a problem. However, there was important morbidity related to inadequate end-organ perfusion and prolonged sedation and mechanical ventilation. We are currently addressing these problems. In the initial phase of the ECMO run we try to optimize off-loading of the heart by paying particular attention to cannula position and decompression of the left side of the heart with early septostomy. We now also try to avoid the complications associated with long-term intensive care such as sedation withdrawal and weakness. Muscle paralysis is avoided whenever possible. Patients are kept comfortable with a morphine infusion of 10 to $40 \mu \mathrm{g} \cdot \mathrm{kg}^{-1} \cdot \mathrm{h}^{-1}$ and, if required, midazolam 1 to $4 \mu \mathrm{g} \cdot \mathrm{kg}^{-1} \cdot \mathrm{min}^{-1}$. Analgesia and sedation are titrated clinically to the lowest dose that keeps the patient awake but comfortable.

The development of mechanical assist devices for children continues to lag far behind that for the adult population, where out-of-hospital support with preservation of end-organ function can be achieved ${ }^{6}$ and sometimes even destination therapy. ${ }^{7}$ Although children can in principle benefit from the same technological advances, there are the additional challenges related to small size. Perhaps more importantly, pediatric VADs have generated little commercial interest because the market is small but paradoxically a large range of device sizes is required.

The use of intra-aortic balloon pump (IABP) counterpulsation is a less invasive mode of support. Although widely used in the adult population, its application has been limited in children. In a study by Hawkins and colleagues, ${ }^{8} 22$ patients received an IABP over a 10-year period with a 59\% survival, and over a similar time period Pennington ${ }^{9}$ reported on 38 patients with a $37 \%$ survival. In children, effective IABP is difficult because of the rapid heart rate. Moreover, the compliance of the aortic wall may result in only slight diastolic augmentation. The small-sized vessels make IABP insertion difficult with high risk of limb ischemia, especially in a low cardiac output state.

There are currently very few prognostic indicators for recovery of myocardial function. Our decision to bridge to recovery is guided mainly by improvement of cardiac function on serial echocardiography during the first few days of mechanical cardiac assistance. The underlying diagnosis may aid the decision making. All patients who were successfully weaned had viral myocarditis. None of the patients with familial dilated cardiomyopathy could be weaned. The problem is that in several patients the diagnosis was not confirmed until some weeks later. Because the time of successful mechanical support is limited to a few weeks, we have a policy of early listing for transplantation. We acknowledge that with this aggressive strategy, some patients may receive cardiac transplantation who could possibly have been bridged to recovery. The other issue is whether the strategy of early high priority listing would disadvantage other patients on the normal transplant waiting list. A recent audit of the United Kingdom pediatric transplant activity has shown that this is not the case. ${ }^{10}$ Despite this, it is of paramount importance that early indicators of myocardial recovery become available. The use of tissue Doppler imaging as a load-independent assessment of myocardial function may hold some promise for the future. ${ }^{11}$

The introduction of $\mathrm{ABO}$ mismatch transplantation has improved the efficiency of use of the donor pool. The exact indications for $\mathrm{ABO}$-incompatible transplantation are still evolving, but we currently believe that infants with antibody titers up to 1:16 can be considered for a mismatch cardiac transplant. The long-term effects of this strategy will only become apparent over time with the maturation of the immune system of the recipients.

\section{Conclusions}

Recent refinements in the management of EDCM in children have significantly reduced early mortality. The timing for initiating mechanical support remains difficult. With the limitations of currently available pediatric devices, the duration of successful support is limited and unnecessary ECMO runs should be avoided. Areas for future research and development include identification of markers of myocardial recovery and the development of mechanical devices for longer term and more physiologic support.

We are grateful to Susan Francis for her commitment, which greatly facilitated the preparation of this manuscript. We thank Lucy Robinson, Jonathan Senior, and Faith Hanstater for their assistance. UK Transplant contributed to the data in this paper.

\section{References}

1. Data UK Transplant Support Service Authority, 1996/1997.

2. Konertz W, Hotz H, Schneider M, Redlin M, Reul H. Clinical experience with the MEDOS HIA-VAD system in infants and children: a preliminary report. Ann Thorac Surg. 1997;63:1138-44.

3. West LJ, Pollock-Barziv SM, Dipchand AI, et al. ABO-incompatible heart transplantation in infants. N Engl J Med. 2001;344:793-800.

4. Kirshbom PM, Bridges ND, Myung RJ, Gaynor JW, Clark BJ, Spray TL. Use of extracorporeal membrane oxygenation in pediatric thoracic organ transplantation. J Thorac Cardiovasc Surg. 2002;123:130-6.

5. Gajarski RJ, Mosca RS, Ohye RG, et al. Use of extracorporeal life support as a bridge to pediatric cardiac transplantation. J Heart Lung Transplant. 2003;22:28-34.

6. Drews TN, Loebe M, Jurmann MJ, Weng Y, Wendelmuth C, Hetzer R. Outpatients on mechanical circulatory support. Ann Thorac Surg. 2003;75:780-5.

7. Heart pump approved for permanent use. FDA consumer. 2003;37:5.

8. Hawkins J, Minich L, Tani L, Pantalos G, Di Russo G, McGough E. Intra-aortic balloon pumping in infants and children. Pediatrics. 1999; S104:664.

9. Pennington GD, Swartz MT. Circulatory support in infants and children. Ann Thorac Surg. 1993;55:233-37.

10. Goldman AP, Cassidy J, de Leval M, et al. The waiting game: Bridging to pediatric heart transplantation. Lancet. In press.

11. Dagianti A, Vitarelli A, Conde Y, Penco M, Fedele F, Dagianti A. Assessment of regional left ventricular function during exercise test with pulsed tissue Doppler imaging. Am J Cardiol. 2000;86(Suppl):30G-2G. 


\section{Discussion}

Dr Charles B. Huddleston (St Louis, Mo). We generally hope that $50 \%$ of patients who require mechanical support will be able to leave the hospital. Your results are obviously much better than that.

There is quite a difference in the presentation and in the manuscript that I received from the abstract that is printed in the program, and it deals with the group I patients. Please comment on what prompted the difference in terms of going back earlier in the time frame in that particular group.

Dr McMahon. Initially we intended to look at a 5-year retrospective study, and then we decided to evaluate 10 years to have a more relevant group.

Dr C. Huddleston. The abstract was accepted on the program with a smaller group. I thought it might be easier too. But that is not important.

Dr McMahon. One of our cardiologists was very keen to look at a decade's worth of treatment.

Dr Huddleston. The hard part with these patients is identifying the ones that are going to get into big trouble or have an arrest before receiving an organ. It is all well and good to have a priority listing established, but I find it extremely difficult to predict those who are going to just teeter on the edge of inotropic support until they get an organ and those who do go on to arrest.

Did you use anything specifically to identify those who were worsening? The manuscript merely includes a comment about those who had hemodynamic deterioration. Were you monitoring them with pulmonary artery catheters, for instance, or any other manipulations in the intensive care unit that really established the presence of a hemodynamic decline?

Having said that, the manuscript seems to indicate that a number of patients did have an episode of cardiopulmonary resuscitation before going on to some sort of mechanical support. Therefore, it was hard for me to tease out that there really was an institution of earlier mechanical support in this second group without there being some objective criteria used in that group to signal the time to go on to support. I find this an extremely difficult issue in our own patient population.

Dr McMahon. Thank you for your comments.

In relation to the question regarding mechanical support and criteria for either being on mechanical support or deteriorating, I think generally everyone finds it very difficult.

With increasing experience within the entire unit, our preference is to keep the patients in house rather than in the referring center, so that they can be monitored for the development of arrhythmias that may further decompensate their heart failure and so that their increasing inotropic requirement can be monitored.

Despite the earlier institution of ECMO in group II, there were a total of 9 patients who had cardiopulmonary resuscitation before ECMO; 4 of them had ventricular tachycardia or a ventricular fibrillatory arrest and 5 had an asystolic arrest.

Even within group II, this is work in process. There were 4 patients who died early in 2000 who would probably have now been kept in house so that mechanical support could have been instituted earlier. I think everyone finds objective criteria quite difficult to pin down.

With regard to your last question about the high priority listing, generally transplantation units within the United Kingdom work together very well. I think the difficulty would be with teenagers who would compete possibly with young adults on the list.

If both pediatric units had a patient on a high priority list, the organ would be offered to the first listed, compatible recipient. However, the units would discuss with each other to decide which of their competing patients was the sickest and generally agree to give the organ to the patient with the highest need. We are proud of the good cooperation between the units in that regard.

Dr Florentino J. Vargas (Buenos Aires, Argentina). Congratulations for your results. Considering how aggressive you have been with the $\mathrm{ABO}$ mismatch, I suppose that you have done something similar regarding weight or size mismatch.

In the pediatric population, in the presence of donor shortage, some groups, including our group, agree to take some significant weight mismatch. We have allowed a weight difference of as much as three times between donor and recipient. Good results were obtained in these patients, the oldest currently being in her eleventh year after transplant. I know some groups would disagree with this policy. Can you comment on that?

Dr McMahon. Thank you. Generally for heart transplants, we accept a weight of two times above the weight of the recipient as the donor organ, and the ABO mismatch transplantation has obviously come into play because of the lack of donor availability in those patients less than 1 year.

Dr Carl L. Backer (Chicago, Ill). In our series of transplantation, we have had a similar improvement in our results.

One of the things that we changed, which I think has helped significantly, is converting from the right atrial technique at the time of the surgical implant to a bicaval technique. At least in our review, that has improved significantly the outcome of our patients. Have you had any change in your surgical technique while treating that cohort.

Dr McMahon. I think I would like to defer that to my senior surgical colleague.

Mr Marc R. de Leval (London, United Kingdom). We have changed to the bicaval technique as well, but much before the change of the management protocol.

I perhaps could add something about medical management. We have appointed a staff cardiologist to look after those patients, and we now have a cardiomyopathy team, which has improved the overall management of those patients, and this includes the timing of putting them on mechanical support.

Dr Richard A. Jonas (Boston, Mass). I notice that you are using ECMO now as your preferred method of mechanical support. How do you decide to decompress the left heart and how you actually do that?

Mr de Leval. All these patients on ECMO undergo a decompression of the left heart, which is done either by blade septectomy or balloon septostomy after perforation of the atrial septum.

Dr Jonas. Do they go to the catheterization laboratory after being put on ECMO?

Mr de Leval. Yes.

Dr Jonas. Am I correct that you do not leave the catheter in? Do you simply make a septostomy or septectomy and allow left-to-right decompression through the atrial septum?

Mr de Leval. That's right.

Dr Jonas. So you are just doing it in everybody? 
Mr de Leval. Yes. That's our protocol; if they are put on ECMO, they have a septectomy.

Dr Carlos J. Troconis (Caracas, Venezuela). In your presentation you pointed out that some of these adults who are connected to VADs or any type of assist device on the left side, at early time, substantially improved and were able to go home, as their status improved from the high priority listing.

Do you think if you approach those babies with DCM in early timing, using a better LVAD, their conditions might improve from the high priority listing, following the same track as in the adults group?

Dr McMahon. I think that was the expectation when the VADs were introduced in the late 1990s, and unfortunately, a high morbidity was encountered. It was just not deemed possible for the program to continue.

That is the overall aim. I would hope that VADs can be produced to allow children to recover with the aid of a VAD outside the intensive care unit.

\section{Availability of Journal back issues}

As a service to our subscribers, copies of back issues of The Journal of Thoracic and Cardiovascular Surgery for the preceding 5 years are maintained and are available for purchase from Mosby until inventory is depleted. Please write to Mosby, Subscription Customer Service, 6277 Sea Harbor Dr, Orlando, FL 32877, or call $800-654-2452$ or $407-345-4000$ for information on availability of particular issues and prices. 